\title{
FORMACIÓN EN LA SOCIEDAD DEL CONOCIMIENTO, UN PROGRAMA DE DOCTORADO CON UNA PERSPECTIVA INTERDISCIPLINAR
}

Resumen: En el curso 2013-2014 se ha puesto en marcha en la Universidad de Salamanca un nuevo Programa de Doctorado en Formación en la Sociedad del Conocimiento, vinculado al Instituto de Investigación "Instituto Universitario en Ciencias de la Educación (IUCE)" de dicha universidad. Más allá de tratarse de un Programa de Doctorado definido bajo el nuevo Real Decreto 99/2011, lo más destacable es la filosofía con la que se ha definido este programa, completamente interdisciplinar, basada en las líneas de investigación, bastante dispares, de los grupos de investigación que soportan en Programa de Doctorado. Estas líneas y grupos complementan diversos enfoques de la Sociedad del Conocimiento, desde la Ingeniería a la Educación, desde la Medicina a la Robótica, o desde los Medios de Comunicación a la Gestión de la Información, pero siempre compartiendo el aprendizaje y las tecnologías como nexo de unión de las diferentes disciplinas que se ven involucradas.

Palabras clave: Sociedad del Conocimiento; Tecnología; Educación; Programa de Doctorado; Interdisciplinaridad.

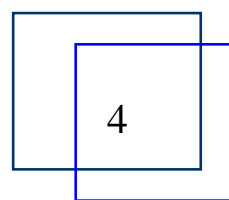




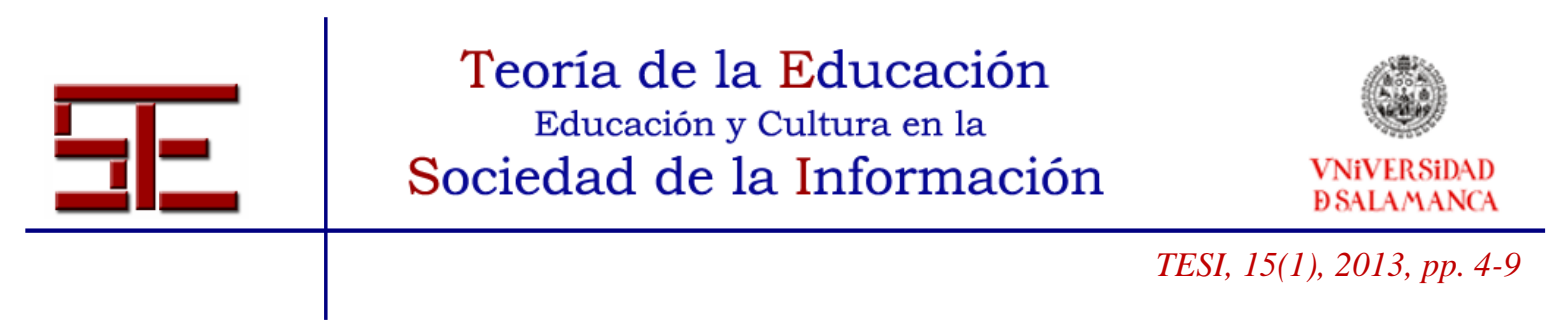

\section{EDUCATION IN KNOWLEDGE SOCIETY, AN INTERDISCIPLINARY PHD PROGRAMME}

Abstract: In the 2013-2014 academic year a new University of Salamanca PhD Programme in Education in Knowledge Society has been defined, which is linked to the Research Institute for Educational Sciences (IUCE) of this University. This is a $\mathrm{PhD}$ Programme defined under the guidelines of RD 99/2011, but its most outstanding characteristic is the philosophy under the Programme has been born, fully interdisciplinary, based on the different research lines of the research groups that support the Programme. These lines and groups complement diverse approaches of the Knowledge Society, from the Engineering to Education, from Medicine to Robotics, or from Communication Media to Information Management, but always sharing learning and technologies as nexus among the different disciplinary approaches that are involved.

Key words: Knowledge Society; Technology; Education; PhD Programme; Interdisciplinary.

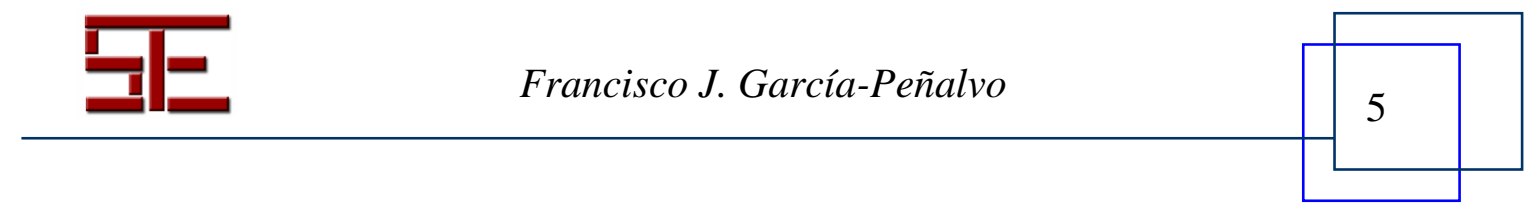




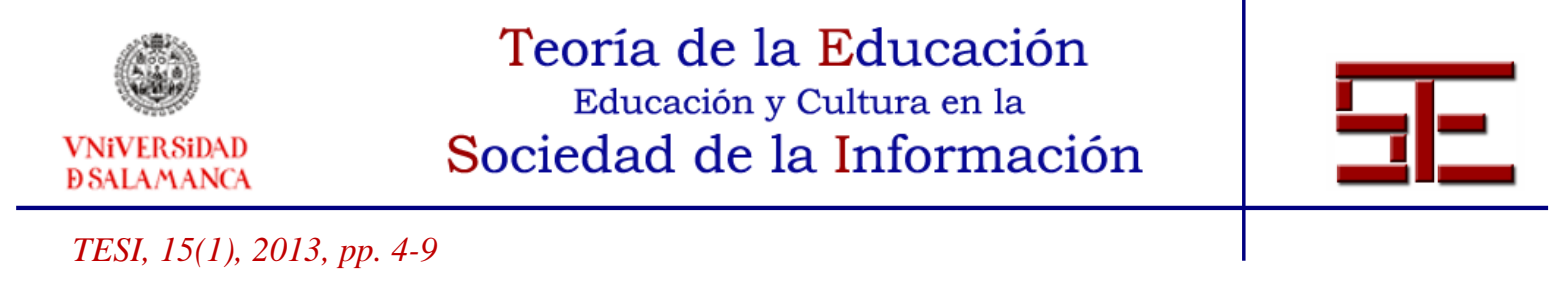

\section{FORMACIÓN EN LA SOCIEDAD DEL CONOCIMIENTO, UN PROGRAMA DE DOCTORADO CON UNA PERSPECTIVA INTERDISCIPLINAR}

Fecha de recepción: 24/10/2013; fecha de aceptación: 19/12/2013; fecha de publicación: 28/02/2014

Francisco J. García-Peñalvo

\section{(fgarcia@usal.es)}

Grupo de Investigación GRIAL, Instituto Universitario de Investigación en Ciencias de la Educación.

Universidad de Salamanca

En el seno del Instituto Universitario de Ciencias de la Educación (IUCEhttp://iuce.usal.es) se ha configurado el Programa de Doctorado en Formación en la Sociedad del Conocimiento (http://www.usal.es/webusal/node/30026, http://knowledgesociety.usal.es/), definido según el Real Decreto 99/2011. Este Programa de Doctorado nace con una clara vocación de presentar los procesos de enseñanza-aprendizaje como auténticos motores de la denominada Sociedad del Conocimiento, para poder disertar y generar nuevo conocimiento en esta línea y bajo una simbiosis con los avances tecnológicos más punteros.

El enfoque de este programa es totalmente interdisciplinar, soportado principalmente por los Grupos de Investigación Reconocidos de la Universidad de Salamanca GRIAL (http://grial.usal.es) (García-Peñalvo, Rodríguez-Conde et al., 2012), GITE (http://gite.usal.es/), OCA (http://campus.usal.es/ oca/), VISUALMED (http://visualmed.usal.es/), Robotics and Society Group (http://gro.usal.es/) y ELECTRA (http://electra.usal.es/), de los cuales GRIAL, GITE y OCA son también Grupos de Excelencia de la Junta de Castilla y León (GR47, GR213 y GR319 respectivamente). Además de estos grupos, se unen al Programa de Doctorado investigadores con líneas de investigación relacionadas con los tópicos del Programa, lo que enriquece el carácter interdisciplinar y multicultural del mismo, al proceder de los ámbitos disciplinares de las Ciencias Sociales (Educación, Comunicación e Información y Documentación), de la Ingeniería y de las Ciencias de la Salud (Medicina). Este grupo de investigadores de la Universidad de Salamanca se ve completado con un amplio plantel de investigadores nacionales e internacionales, que conforma la vanguardia de las líneas de investigación relacionadas con la Formación en la Sociedad del Conocimiento que, sin pretender ser exhaustivos, cubren los siguientes grandes descriptores o áreas de investigación:

•Evaluación Educativa y Orientación.

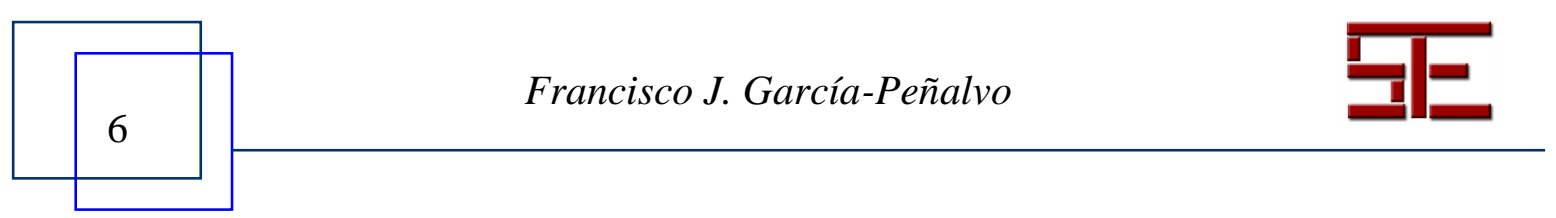




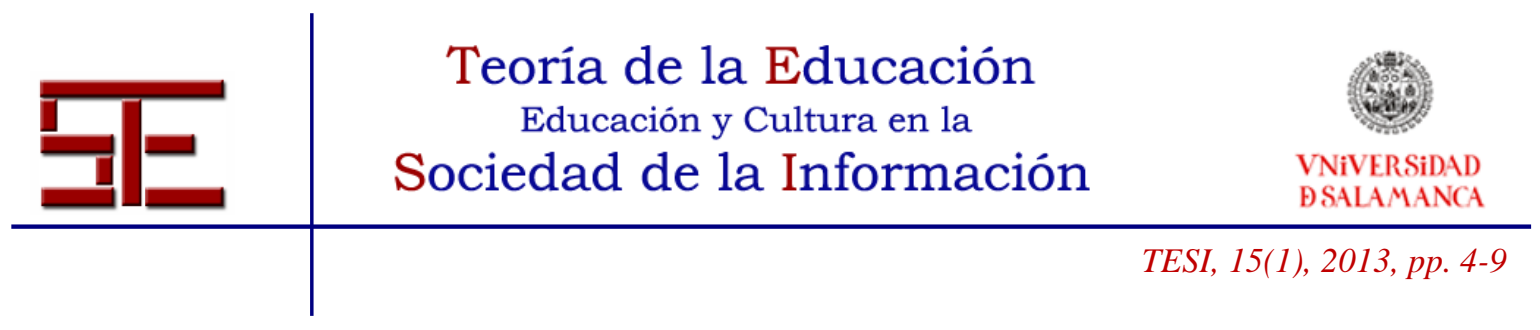

-Interacción y eLearning.

-Investigación-Innovación en Tecnología Educativa.

-Medios de Comunicación y Educación.

-Medicina y Educación.

-Robótica educativa.

-Ingeniería y Educación.

•Educación y Sociedad de la Información.

Este Programa de Doctorado ofrece un marco de trabajo para que se den las sinergias y las relaciones simbióticas entre los recursos intelectuales provenientes de la práctica totalidad de las ramas del conocimiento de la Universidad con el fin último de elevar la Educación como uno de los derechos fundamentales y motor de la sociedad actual, a la par que se tienden los puentes necesarios para afrontar, de una forma reflexiva, colaborativa e interdisciplinar, los importantes retos que se relacionan, por tanto, con el progreso de la humanidad.

Mediante el Programa de Doctorado en Formación en la Sociedad del Conocimiento, se intenta capacitar a sus futuros doctores no solo en las competencias disciplinares, de conocimiento teórico y práctico o de reflexión y pensamiento crítico que cualquier Programa de Doctorado compartiría como elementos básicos, sino que además se quiere transmitir una perspectiva de encarar la resolución de los complejos problemas de nuestra sociedad actual que compagine, por un lado, la interdisciplinaridad y, por otra parte, la apuesta por el Conocimiento en Abierto (García-Peñalvo, García de Figuerola, \& Merlo, 2010a, 2010b). Todo ello como fiel reflejo de cómo pensamos que la Universidad debe avanzar para liderar la formación y transición hacia la Sociedad del Conocimiento (Berlanga, García-Peñalvo, \& Sloep, 2010; García-Peñalvo, 2011), a la par que sus egresados (doctorados en este caso y, por tanto, egresados con el máximo nivel de capacitación, madurez y capacidad de liderazgo) deben ser capaces de transmitir esta perspectiva, en el ejercicio de su actividad profesional, al tejido productivo y a la sociedad en general, estando sobradamente capacitados para conjugar esa formación formal e informal recibida (García-Peñalvo, Colomo-Palacios, \& Lytras, 2012).

La aceptación que ha tenido este Programa de Doctorado en su primera edición se podría calificar como de excelente. Sobre unas previsiones de 25 plazas en el curso 2013-2014, se han recibido un total de 36 solicitudes, de las cuales finalmente la Comisión Académica del Programa de Doctorado ha aceptado 29, a las que se les ha impuesto un plan de trabajo que les posibilite el seguir adscritos al Programa de Doctorado durante el periodo estimado de 3 o 5 años (en función de si son doctorados a

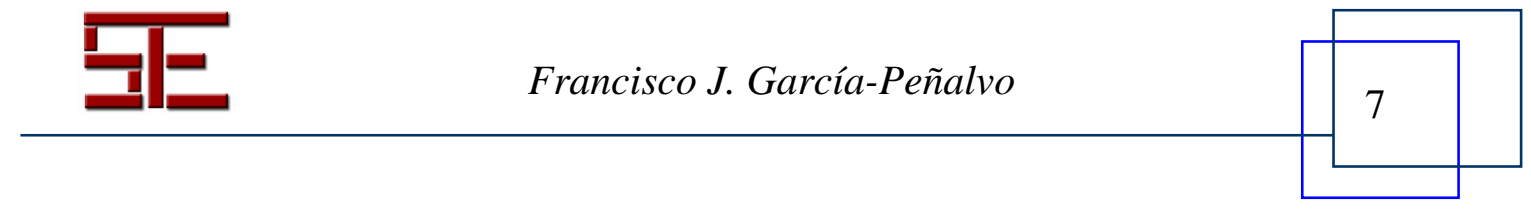




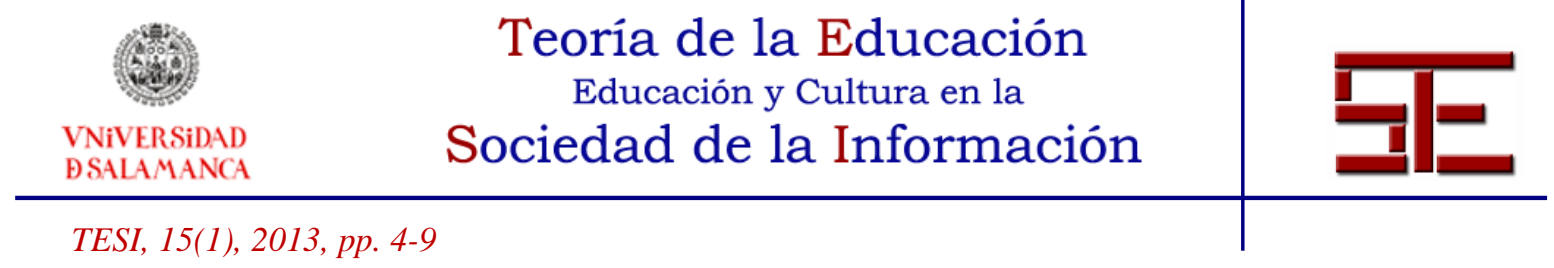

tiempo completo o tiempo parcial) en el que tienen que finalizar el desarrollo de sus tesis doctorales.

De forma paralela a la definición de este Programa de Doctorado, se ha puesto en marcha una Conferencia Internacional, Technological Ecosystems for Enhancing Multiculturality (TEEM - http://teemconference.eu/), cuyos tópicos de interés coinciden con el enfoque y líneas de investigación del Programa de Doctorado, y que tiene, precisamente, el objetivo de construir una comunidad de investigación internacional en esta área de interés.

En este Congreso los doctorandos y los investigadores del Programa de Doctorado en Formación del Conocimiento siempre encontrarán un espacio en el que compartir sus avances, bien en sus sesiones temáticas, bien en su Consorcio Doctoral. Como resultado de la primera edición de este evento, TEEM 2013 (http://teemconference.eu/2013) (García-Peñalvo, 2013; García-Peñalvo, García-Holgado, \& Cruz-Benito, 2013), celebrado en Salamanca del 14 al 16 de noviembre de 2013, se ha invitado a un conjunto representativo de autores a que extiendan sus contribuciones que, tras ser nuevamente revisadas por miembros del Comité de Programa, han sido aceptadas para publicarse en este número de la revista Teoría de la Educación: Educación y Cultura en la Sociedad de la Información (TESI).

Concretamente, $\mathrm{M}^{\mathrm{a}}$ Goretti Alonso de Castro, coordinadora del Área de Evaluación y Control del Organismo Autónomo Programas Educativos Europeos, en su artículo "Educational projects based on mobile learning", hace un repaso por algunos de los proyectos europeos más significativos relacionados con el mLearning. Siguiendo con esta temática de la movilidad y el aprendizaje, Sánchez Prieto et al., en su artículo "Understanding mobile learning: devices, pedagogical implications and research lines", realizan un repaso del estado del arte del mLearning así como de sus tendencias actuales desde una perspectiva de investigación educativa. Sin abandonar el uso de los dispositivos móviles con fines educativos, Ana Pérez Escoda, en su artículo "Media literacy in primary school: new challenges in the digital age", presenta los resultados del proyecto Conectados, un proyecto de alfabetización digital en el aula que ha utilizado una herramienta específica, el iPad, con el objetivo de mejorar las competencias digitales de los niños a partir de 7-8 años de edad, que son, ya, usuarios de Internet. Amo et al. en su artículo "Approaches for quality in pedagogical and design fundamentals in MOOCS", abordan el controvertido y apasionante mundo de los cursos masivos desde una perspectiva pedagógica. Por último, Patricia Torrijos Fincias y Juan Francisco Martín Izard, en su artículo "Desarrollo de competencias emocionales en el profesorado de educación secundaria a través de una intervención por programas", abordan el desarrollo de la competencia emocional en el profesorado de secundaria como estrategia para hacer frente a los desafíos de su práctica diaria, y prevenir el

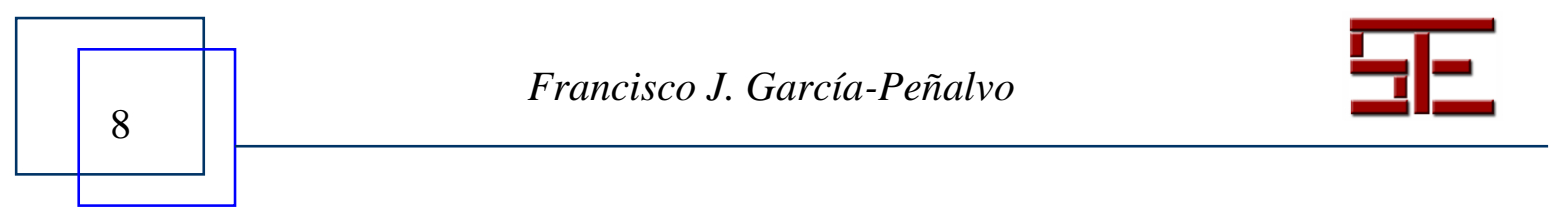




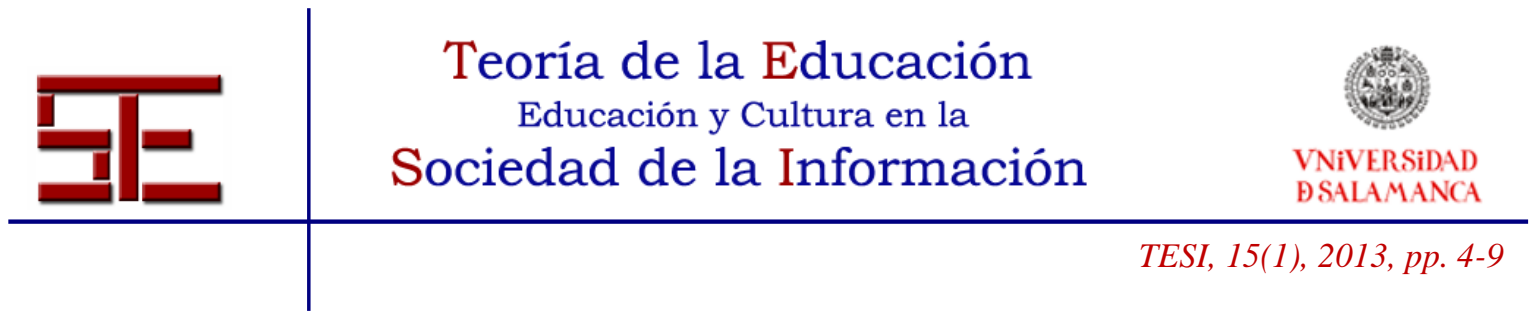

malestar, estrés y sentimientos de frustración que pueden aparecer ante las demandas y conflictos por parte de padres, estudiantes, otros compañeros y equipo directivo.

\section{REFERENCIAS}

Berlanga, A. J., García-Peñalvo, F. J., \& Sloep, P. B. (2010). Towards eLearning 2.0 University. Interactive Learning Environments, 18 (3), 199-201. doi: 10.1080/10494820.2010.500498.

García-Peñalvo, F. J. (2011). La Universidad de la próxima década: La Universidad Digital. En C. Suárez-Guerrero \& F. J. García-Peñalvo (Eds.), Universidad y Desarrollo Social de la Web (pp. 181-197). Washington DC, USA: Editandum.

- (2013). Proceedings of the First International Conference on Technological Ecosystem for Enhancing Multiculturality, TEEM 2013. New York, USA: ACM.

García-Peñalvo, F. J., Colomo-Palacios, R., \& Lytras, M. D. (2012). Informal learning in work environments: training with the Social Web in the workplace. Behaviour \& Information Technology, 31 (8), 753-755. doi: 10.1080/0144929x.2012.661548.

García-Peñalvo, F. J., García de Figuerola, C., \& Merlo, J. A. (2010a). Open knowledge management in higher education. Online Information Review, 34 (4), 517-519.

- (2010b). Open knowledge: Challenges and facts. Online Information Review, 34 (4), 520-539. doi: 10.1108/14684521011072963.

García-Peñalvo, F. J., García-Holgado, A., \& Cruz-Benito, J. (2013). Proceedings of the TEEM'13 Track on Knowledge Society Related Projects. Salamanca, Spain: Grupo GRIAL.

García-Peñalvo, F. J., Rodríguez-Conde, M. J., Seoane-Pardo, A. M., Conde-González, M. Á., Zangrando, V. \& García-Holgado, A. (2012). GRIAL (GRupo de investigación en InterAcción y eLearning), USAL. IE Comunicaciones. Revista Iberoamericana de Informática Educativa (15), 85-94.

Para citar el presente artículo puede utilizar la siguiente referencia:

García-Peñalvo, F. J. (2014). Formación en la sociedad del conocimiento, un programa de doctorado con una perspectiva interdisciplinar. Revista Teoría de la Educación: Educación y Cultura en la Sociedad de la Información. 15(1), 4-9 [Fecha de consulta: $\mathrm{dd} / \mathrm{mm} / \mathrm{aaaa}]$.

http://campus.usal.es/ revistas_trabajo/index.php/revistatesi/article/view/11561/11981

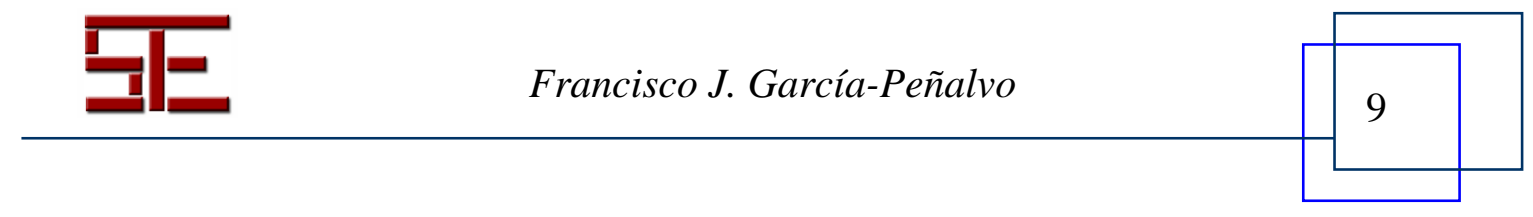

